\title{
Hydrophobic Silica Aerogels by Silylation
}

\author{
Yannan Duan ${ }^{\mathrm{a}}$, Sadhan C. Jana, ${ }^{\mathrm{a},{ }^{*}}$, Bimala Lama ${ }^{\mathrm{b}}$, Matthew P. Espe ${ }^{\mathrm{b}}$ \\ ${ }^{a}$ Department of Polymer Engineering, The University of Akron, Akron, Ohio, 44325-0301, USA \\ ${ }^{\mathrm{b}}$ Department of Chemistry, The University of Akron, Akron, Ohio, 44325-3601, USA. \\ *Corresponding author at janas@uakron.edu
}

Keywords: silica aerogel, hydrophobic, surface modification

\begin{abstract}
In this work, silica gel networks were modified with a silylating agent dimethoxy-methyl (3,3,3-trifluoropropyl) silane (SiF3) to obtain hydrophobic aerogels of various surface energy values. The baseline aerogels were synthesized from tetraethoxy silane (TEOS) using a twostep sol-gel process followed by supercritical drying in liquid carbon dioxide. The resultant aerogels were characterized using scanning electron microscopy, Instron tensile tester, contact angle goniometry, and nitrogen adsorption-desorption isotherms. Three modification methods were studied. In method 1 , TEOS and SiF3 were combined before gelation; in method 2, SiF3 was added after TEOS was hydrolysed and before its condensation, and in method 3, SiF3 was added after the gels were produced from TEOS. It was found that method 3 produced the best results in terms of achieving high values of hydrophobicity and compressive properties. The data on solid state ${ }^{13} \mathrm{C}$ and ${ }^{29} \mathrm{Si}$ NMR spectra revealed chemical reactions between the silylating agents and the silanol groups on silica surface. The bulk density and the fractal dimensions of silica networks gleaned from small angle X-ray scattering (SAXS) data showed weak dependence on the degree of silylation. The silylation process rendered the aerogels strongly hydrophobic and also doubled its compressive modulus.
\end{abstract}




\section{Introduction}

Aerogels are highly porous solid materials derived from gels by replacing the liquid inside the pores by air $^{1}$. The solid particle networks and associated pores in the gels survive the capillary stress if the liquid is removed under supercritical conditions. Silica aerogels are a unique class of highly porous materials (porosity $>95 \%$ ) and offer the following properties: low density $\left(3-350 \mathrm{mg} / \mathrm{cm}^{3}\right),{ }^{2,3}$ large surface area $\left(500-1200 \mathrm{~m}^{2} / \mathrm{g}\right)$, low thermal conductivity (ranging from 0.004 to $0.03 \mathrm{~W} / \mathrm{m} \cdot \mathrm{K}$ ), low dielectric constant $(1.1-2.2){ }^{4,5}$ low index of refraction $(\sim 1.05),{ }^{6}$ and an array of optical appearances such as transparency, ${ }^{7,8}$ opacity, or translucence. ${ }^{9}$ These interesting properties make silica aerogels suitable for applications in high-energy particle physics (Cherenkov emitters). ${ }^{10}$ Monolithic silica aerogels are well suited to deal with the energy savings coupled with alleviating some of the environmental concerns, ${ }^{11}$ e.g., transparent and super-insulating double windows, ${ }^{12}$ or promising space science application. ${ }^{13}$ Additionally, silica aerogels can be used as adsorbents, e.g. for oils and organic liquids, or used as sensors, ${ }^{14}$ catalysts, ${ }^{15}$ storage media, and templates. ${ }^{16}$ A number of applications of silica aerogels in life sciences are emerging, such as in biocatalysis ${ }^{17}$ with a lipase enzyme or in a process to detect a viral particle by immobilized bacteria. ${ }^{18}$ Despite these applications, fragility at low mechanical stress and hygroscopic nature are two deterrents to more widespread applications.

It is believed that a few Si-O-Si bonds in the "neck" regions hold the neighbouring spherical secondary silica particles together. ${ }^{19}$ Consequently, much focus is given to the neck regions for reinforcement of silica based aerogels. ${ }^{20-22}$ Various approaches have been exploited by building extra covalent bonds in the "neck" regions using small molecules, polymers, and hybrid nanoparticles. Leventis and coworkers ${ }^{19,23,24}$ reported mechanically reinforced silica 
aerogels through the reaction of isocyanates with the residual hydroxyl groups on the secondary silica particles. Duan et al. reported significant improvements in compressive modulus and hydrophobicity by reacting silica particles with 1-3 nm size polyhedral oligomeric silsesquioxane (POSS) molecules ${ }^{25}$ and by using silane-terminated polyurethanes ${ }^{26}$ as a co-precursor with small changes in bulk density.

To alter the hygroscopic nature of silica aerogels and to make them suitable for more widespread applications, surface modification of silica particles with silane-containing organic groups was considered in this work. Previous work showed that moderately hydrophobic ${ }^{27}$ and even super-hydrophobic ${ }^{28}$ silica aerogels can be obtained by aging the alcogels in solution of halogen-containing silanes; alkyl-alkoxy silanes with chlorine atoms are the most commonly used. ${ }^{27,}{ }^{28}$ Water contact angle values were measured by placing water droplets directly on the aerogel surfaces and, therefore, reflected the influence of trapped air inside the mesoporous structures. However, surface modification alleviates the concerns related to the collapse of silica networks during drying and particle network coarsening due to reactions between adjacent silica particles. Therefore, it is critical to understand the impact of silylating agent and incorporation process on the final morphology and properties of aerogels. A more thorough review on synthesis of hydrophobic silica aerogels is presented elsewhere ${ }^{29}$.

In this work, a silane molecule with trifluoropropyl substitution - dimethoxy-methyl $(3,3,3$ trifluoropropyl) silane, denoted as $\mathrm{SiF}_{3}$, was used to modify the silica alcogels. It was expected that this silylating agent would diffuse into the small pores of silica alcogels and eliminate the residual $\mathrm{Si}-\mathrm{OH}$ groups by silylating reactions. As a consequence, the process would render the surfaces of the silica particles hydrophobic and, in addition, might produce mechanical reinforcement. It was anticipated at the outset that such treatment would not exert 
significant effects on morphology and density. The study answered several questions: What should be an appropriate scheme for introduction of $\mathrm{SiF}_{3}$ molecules into silica networks? How much $\mathrm{SiF}_{3}$ can enter into silica network? Can $\mathrm{SiF}_{3}$ molecules produce mechanical reinforcement, much the same way as coarsening of silica networks? Silica networks are known to have fractal structures. $\mathrm{Do} \mathrm{SiF}_{3}$ molecules alter the fractal nature of silica particles or the pore structures or the density? What are the effects of silylation on surface energy of silica particles? Prior work ${ }^{28}$ reported super hydrophobic aerogels by treating silica particles with TMCS and HMDZ, although the changes of polarity of silica particles due to such treatment were not reported. Moreover, it is evident that measurement of water contact angle on aerogel surface would be strongly influenced by air trapped inside the mesoporous structures. Such measurements would not yield surface energy data as function of chemical treatment of silica particles.

\section{Experimental}

\subsection{Materials.}

The silica particles network was derived from tetraethoxy silane (TEOS) ${ }^{27,28}$. Dimethoxymethyl (3,3,3-trifluoropropyl) silane (denoted as $\mathrm{SiF}_{3}$ ) was selected due to its high fluorine content and higher surface energy compared to chlorine atoms. All solvents and reagents were used as received from the manufacturers. TEOS, nitric acid (70\%), and ammonium hydroxide solution (28.0-30.0\% $\mathrm{NH}_{3}$ basis) were purchased from Sigma Aldrich. Reagent grade ethanol was purchased from Fisher Scientific. Dimethoxy-methyl (3,3,3trifluoropropyl) silane (97.0\% GC grade) was purchased from Gelest Inc. (Morrisville, PA).

\subsection{Preparation of silica aerogels modified with $\mathrm{SiF}_{3}$ silane.}


The synthesis of aerogels involved two steps: (1) preparation of the alcogel and (2) removal of the solvent from the alcogels via supercritical drying. The alcogels were prepared by a two-step acid-base catalyzed sol-gel process. Solution A, containing $22.30 \mathrm{~mL}$ TEOS, 7.20 $\mathrm{mL}$ of water $(7.20 \mathrm{~g})$ and $30.50 \mathrm{~mL}$ ethanol, was hydrolyzed under acidic conditions using nitric acid $(\mathrm{pH} \sim 2)$. The mixture was sealed and stirred by a stir bar for $1 \mathrm{~h}$ and then cooled in a dry ice-acetone bath. Solution $\mathrm{B}$, which contained $5.40 \mathrm{~mL}$ water, $34.60 \mathrm{~mL}$ ethanol, and a base catalyst ammonium hydroxide solution $(\mathrm{pH}$ ), was cooled in dry ice-acetone bath (around $-65{ }^{\circ} \mathrm{C}$ ) to control premature gelation when combined with solution A. The total molar ratio of TEOS to $\mathrm{H}_{2} \mathrm{O}$ was maintained at 1:7. Gels were formed in polypropylene mold of $12.5 \mathrm{~mm}$ diameter. Solutions A and B were vigorously mixed, poured into the molds, and allowed to gel and age for 24 hours. The acidic condition during hydrolysis of TEOS and the basic condition during condensation of the silanes provided high rates of reactions.

Modification of silica particles using $\mathrm{SiF}_{3}$ was carried out using three methods - (1) TEOS and $\mathrm{SiF}_{3}$ were mixed together in solution A prior to hydrolysis and condensation by mixing with solution $\mathrm{B}$, (2) $\mathrm{SiF}_{3}$ was mixed with solution A after TEOS was completely hydrolyzed and before adding solution $\mathrm{B}$, and (3) $\mathrm{SiF}_{3}$ was added to alcogel and allowed to react with silica particles. A screening study was conducted to determine an appropriate scheme of inclusion of $\mathrm{SiF} 3$ following methods 1,2 , or 3 . The preparation process and composition of each type of aerogels are summarized in Table 1. All these aerogels were prepared by a twostep acid-base catalyzed sol-gel process, and the concentration of TEOS was kept the same at $1 \mathrm{~mol} / \mathrm{L}$ for these five compositions. The amounts of SiF3 used to modify the TEOS-based aerogels are also listed in Table 1. Sample No. 1 was prepared as a control material without modification by SiF3. In method $1,4.0 \mathrm{~g}$ and $5.0 \mathrm{~g}$ of $\mathrm{SiF} 3$ were mixed with $6.250 \mathrm{~g}(6.70$ $\mathrm{mL}$ ) TEOS for sample No. 2 and 3 respectively and hydrolyzed at $\mathrm{pH} 2$. The mixtures were then allowed to condense using the base catalyst. In preparation of sample No. 4 by method 
2, $6.250 \mathrm{~g}(6.70 \mathrm{~mL})$ TEOS was pre-hydrolyzed at $\mathrm{pH} 2$ and $4.0 \mathrm{~g}$ of $\mathrm{SiF} 3$ was added along with the base catalyst. This allowed the hydrolyzed species to condense and form alcogels as soon as $\mathrm{SiF} 3$ and base catalyst were added. In the last modification process (sample No. 5, method 3), the alcolgels were prepared by following the same procedure as in sample No. 1 and the gels were soaked in ethanol solution of $5.0 \mathrm{~g} \mathrm{SiF} 3$. The gels were allowed to age in the solution for 5 days, solvent exchanged with ethanol, and finally dried using supercritical carbon dioxide.

It will be shown later that method 3 produced the best results among the three methods. In view of this, $\mathrm{SiF}_{3}$-modification by method 3 was more extensively studied. After the gels were formed, different weight percent of $\mathrm{SiF}_{3} /$ ethanol solution $(5 \mathrm{wt} \%, 10 \mathrm{wt} \%, 15 \mathrm{wt} \%, 20$ $\mathrm{wt} \%, 25 \mathrm{wt} \%$ ) were prepared and ten alcogel specimens were soaked in each solution for 3 days. The wet gels were rinsed with ethanol 4 times and supercritically dried in an autoclave at $55{ }^{\circ} \mathrm{C}$ and $10 \mathrm{MPa}$ pressure using liquid $\mathrm{CO}_{2}$. The expected reactions and the final structures of the silica networks are schematically presented in Figure 1. These series of aerogels are denoted as "T-SiF3-wt\%", e.g. T-SiF3-5wt\% represents TEOS-based aerogels soaked in $5 \mathrm{wt} \% \mathrm{SiF}_{3} /$ ethanol solution. The unmodified aerogel is denoted as $\mathrm{T}-0$. 
Table 1.

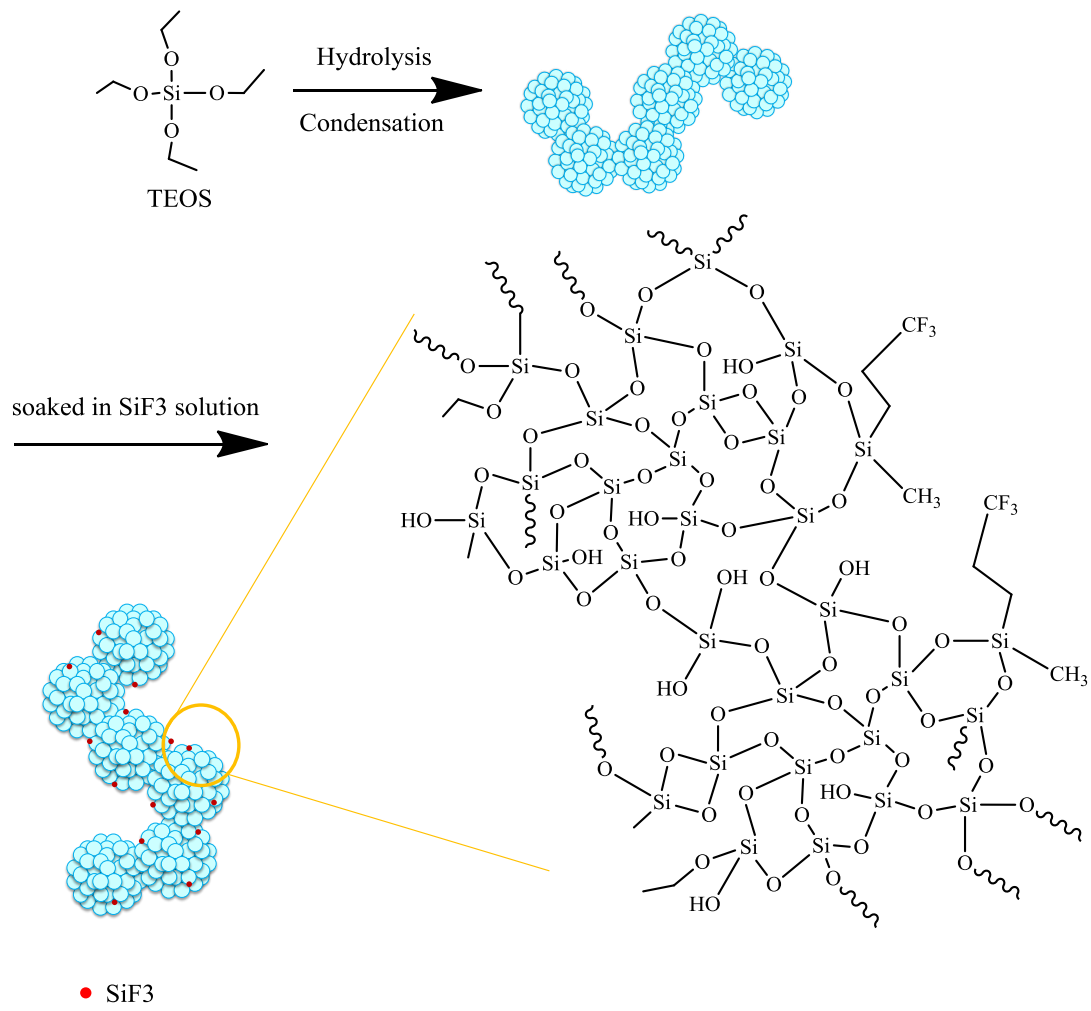

Figure 1. Structure of silica networks modified with $\mathrm{SiF}_{3}$ by method 3 .

Composition and contact angle data of TEOS-based aerogels as function of different modification steps. The contact angle data was obtained by placing liquid drops on compressed discs of aerogels.

\begin{tabular}{ccccc}
\hline $\begin{array}{c}\text { Sample } \\
\text { No. }\end{array}$ & $\begin{array}{c}\text { Modification } \\
\text { process }\end{array}$ & $\begin{array}{c}\text { weight of SiF3 } \\
\text { added }(\mathrm{g})\end{array}$ & $\begin{array}{c}\text { Contact angle } \\
\text { with } \mathrm{H}_{2} \mathrm{O}\end{array}$ & $\begin{array}{c}\text { Contact angle with } \\
\mathrm{CH}_{2} \mathrm{I}_{2}\end{array}$ \\
\hline 1 & TEOS w/o $\mathrm{SiF}_{3}$ & none & $27.5 \pm 0.3^{\circ}$ & $25.7 \pm 0.4^{\circ}$ \\
2 & Method 1 & 4.0 & $106.0 \pm 0.6^{\circ}$ & $51.5 \pm 1.0^{\circ}$ \\
3 & Method 1 & 5.0 & $134.0 \pm 1.0^{\circ}$ & $85.9 \pm 0.6^{\circ}$ \\
4 & Method 2 & 4.0 & $70.6 \pm 0.9^{\circ}$ & $38.6 \pm 0.6^{\circ}$ \\
5 & Method 3 & 5.0 & $142.2 \pm 0.8^{\circ}$ & $106.1 \pm 0.8^{\circ}$ \\
\hline
\end{tabular}

\subsection{Characterization.}


Bulk density was obtained from mass and volume of aerogel specimens. The diameter and length of aerogel specimen were measured by a calliper with a precision of $0.01 \mathrm{~mm}$. Three measurements for each specimen and five specimens for each type of aerogels were used to obtain reproducible data. Shrinkage in diameter was calculated by comparing the diameter of the gel and the aerogel.

Solid state ${ }^{13} \mathrm{C}$ and ${ }^{29} \mathrm{Si}$ nuclear magnetic resonance (NMR) spectra were used to determine the extent of hydrolysis and condensation reactions of the silanes and the reactions between the silanes and $\mathrm{SiF}_{3}$ molecules. ${ }^{29}$ The data were collected on a Varian INOVA $400 \mathrm{MHz}$ (9.4T) spectrometer using a Varian $4 \mathrm{~mm}$ DR-T3 probe.

Small angle X-ray scattering (SAXS) is a choice method for studying the structure of porous materials in 1-100 $\mathrm{nm}$ range. ${ }^{31}$ SAXS was used as a tool for determining the mass fractal features of silica aerogels. ${ }^{28,32,33}$ The fractal dimension is measured from the power-decay of the static structure in the Porod region, ${ }^{33}$ as given in equation (1):

$$
\lim _{q \rightarrow \infty}\left[I(q) q^{(6-D s)}\right]=P(1)
$$

where $I(q)$ is the SAXS intensity at low angles as a function of the modulus of the scattering vector $q=\frac{4 \pi \sin \theta}{\lambda}, 2 \theta$ being the scattering angle, $\lambda$ the $\mathrm{X}$-ray wavelength, Ds is the dimensionality of the fractal and $\mathrm{P}$ is a constant. A value of Ds $=2$ corresponds to classical (two-dimensional) surfaces. Several gels were shown to exhibit interfaces having the fractal properties with $2<$ Ds $<3$, which has a slope between -3 and $-4,{ }^{34}$ describing the elementary units as $3 \mathrm{D}$ particles with fractal rough surfaces. ${ }^{35}$ 
The SAXS intensity was collected using an X-ray generator of Rigaku MicroMax-002+ and $\mathrm{Cu}$ as the X-ray source with a wavelength of $1.54 \AA$. The voltage was set at $45 \mathrm{kV}$ with a current of $0.88 \mathrm{~mA}$. The scattering signal was detected by SAXS legacy $120 \mathrm{~mm}$ detector. The range of angle was selected by setting scattering vector $q$ in the range of $0.017-0.32 \AA^{-1}$.

The compressive modulus of aerogels was determined from the stress-strain data obtained using a tensile tester, Instron 5567, Canton, MA following ASTM D695-85 method for compressive test. The ASTM standard calls for a slenderness ratio of 11:1 to 16:1, however, using this sample size would have caused some of the lower density specimens to buckle. Cylindrical samples of diameter approximately $12 \mathrm{~mm}$ and height approximately $15 \mathrm{~mm}$ were used. Each aerogel cylinder was sanded at the top and bottom to ensure that they were smooth and level. At least five cylinders were tested for each specimen. The crosshead speed for the test is chosen at $1.27 \mathrm{~mm} / \mathrm{min}$.

The morphology of aerogels was characterized by scanning electron microscopy (SEM). For this purpose, the aerogels were fractured at room temperature and SEM images were taken after sputter coating the fractured surface with silver. SEM images were taken using Scanning Electron Microscope (SEM, JEOL JSM5310).

The surface area, average pore size, and pore size distribution were measured via gas adsorption measurement by studying the adsorption and desorption isotherms. The surface area, pore size, and pore size distribution were measured using TriStar II from Micromeritics instrument. Each sample was cut and placed in the chamber followed by outgassing either at $40{ }^{\circ} \mathrm{C}$ or at room temperature until the mass attained a constant value. Adsorption/desorption isotherms were collected by using nitrogen as the adsorbent at $-196{ }^{\circ} \mathrm{C}$ using liquid nitrogen bath to achieve this temperature. 
The surface properties were studied by measuring the contact angle of water and diiodomethane. Aerogel specimens were converted into compressed solid discs to eliminate the effect of pore structures following a test method described elsewhere. ${ }^{25}$ For each disc, 10 15 measurements were taken to obtain reproducible results. The contact angle values were calculated from the image taken by Rame-Hart contact angle goniometer (model 100-00) on both edges of the drop using the software, ImageJ 1.42. The surface energy was calculated using a procedure described elsewhere. ${ }^{25}$

\section{Results and discussion}

\subsection{Appropriate scheme of silica particle modification}

The surface modification of silica gels by $\mathrm{SiF}_{3}$ molecules were expected to alter the hydrophilic nature of the aerogels. The post-gelation process (method 3) for surface modification by $\mathrm{SiF}_{3}$ molecules was adopted in this work for two primary reasons - (1) the modification process should not alter the morphology of silica particles and (2) the modification process should not be impacted by diffusional limitations.

One may argue that the inclusion of $\mathrm{SiF}_{3}$ with TEOS in the starting formulation (method 1) should be more useful in integrating $\mathrm{SiF}_{3}$ molecules to silica networks. Note that the two methoxy groups in $\mathrm{SiF}_{3}$ molecules are capable of undergoing hydrolysis and condensation reactions like the ethoxy groups in TEOS. The inclusion of $\mathrm{SiF}_{3}$ in the starting formulation (method 1) may lead to a situation where these molecules may remain trapped inside the secondary particles. The contact angle data on the compressed discs of these aerogels with various modifications are shown in Table 1.

The compressed disc of aerogels without modification by $\mathrm{SiF}_{3}$ gave a contact angle of $27^{\circ}$ with deionized water and $26^{\circ}$ with diiodomethane. The aerogels modified with $\mathrm{SiF}_{3}$, 
irrespective of the modification process, showed significant increase in contact angle with deionized water and diiodomethane, suggesting that $\mathrm{SiF}_{3}$ is an effective molecule for surface modification. However, a glance at the data presented in Table 1 gives us a hint that method 3 offers more effective surface modification. The comparison of data for sample No. 2 and 4 suggests that method 1 is better than method 2. In method $1, \mathrm{SiF}_{3}$ underwent hydrolysis along with TEOS under acidic condition, and the hydrolyzed species condensed as the condition was changed to basic. In method 2, TEOS was pre-hydrolyzed, and condensed very fast under basic condition. This did not give enough time for $\mathrm{SiF}_{3}$ molecules to hydrolyze and cocondense with hydrolyzed TEOS. Note that alkoxy silanes hydrolyze slowly under basic conditions. Consequently, in this case, the primary and secondary silica particles were formed primarily from TEOS by the time appreciable hydrolysis of SiF3 molecules occurred under basic condition. Thus, the reactions between hydrolyzed TEOS and $\mathrm{SiF}_{3}$ might not be as effective as in pre-hydrolysis process in method I. This offers an explanation of the lower contact angle value of only $71^{\circ}$ with deionized water for materials prepared by method 2 . The comparison of sample No. 3 and 5 suggests that method 3 is more effective than method 1. There exists a possibility that some $\mathrm{SiF}_{3}$ molecules remain hidden inside the secondary particles instead of coating the surfaces in method 1 . The $\mathrm{SiF}_{3}$ molecules can only age on the surfaces of the secondary particles via silylation reaction for post-gelation process in method 3, as molecular diffusion limits ingress of $\mathrm{SiF} 3$ molecules inside the secondary particles. Based on these results, the post-gelation approach in method 3 is selected in the rest of the paper as a means of more effective surface modification. It is believed that, in this manner, $\mathrm{SiF}_{3}$ molecules eliminate the residual $-\mathrm{OH}$ groups on silica particle surfaces in a reasonable reaction period.

\subsection{Density, shrinkage, and mechanical properties}


The baseline silica aerogel and the aerogels modified with different amounts of $\mathrm{SiF}_{3}$ were found to be opaque as seen in Figure S1. The values of density and the diameter shrinkage of all aerogels are listed in Table 2. The data in Table 2 show small (less than $10 \%$ ) increases in density in SiF3-modified specimens. The silylation scheme adopted in this work, therefore, caused only small changes in density. The diameter shrinkage gradually reduced with an increase of the amount of $\mathrm{SiF} 3$ used in silylation reactions. It is noted that the percentage of shrinkage reduced from $7.2 \%$ for unmodified aerogels to $2.8 \%$ for aerogel modified with 25 wt $\%$ solution of $\mathrm{SiF}_{3}$.

The compressive modulus increased weakly with $\mathrm{SiF}_{3}$ concentration up to $10 \mathrm{wt} \%$, showed a large increase for $15 \mathrm{wt} \%$, and remained insensitive to further increases in $\mathrm{SiF}_{3}$ concentration. It is apparent from Table 2 that the compressive modulus doubled with less than $10 \%$ increase in density when the gels were treated with $15 \mathrm{wt} \%$ or higher concentration of $\mathrm{SiF}_{3}$ solution. This large increase in compressive modulus may be attributed to $\mathrm{SiF}_{3}$ molecules potentially bridging all neighbouring secondary particles. This scenario will be examined further in the context of silica particle morphology, surface area, and fractal dimensions of silica networks.

Table 2. Density, diameter shrinkage, and compressive modulus of $\mathrm{SiF}_{3}$-modified aerogels.

\begin{tabular}{lccc}
\hline \multicolumn{1}{c}{ Specimen } & Density $(\mathrm{g} / \mathrm{cm} 3)$ & Shrinkage $(\%)$ & Compressive modulus $(\mathrm{MPa})$ \\
\hline $\mathrm{T}-0$ & $0.119 \pm 0.003$ & $7.2 \pm 2.2$ & $0.62 \pm 0.04$ \\
$\mathrm{~T}-\mathrm{SiF} 3-5 \mathrm{wt} \%$ & $0.123 \pm 0.007$ & $6.6 \pm 2.2$ & $0.67 \pm 0.06$ \\
$\mathrm{~T}-\mathrm{SiF} 3-10 \mathrm{wt} \%$ & $0.121 \pm 0.004$ & $4.7 \pm 2.0$ & $0.79 \pm 0.06$ \\
$\mathrm{~T}-\mathrm{SiF} 3-15 \mathrm{wt} \%$ & $0.125 \pm 0.002$ & $4.2 \pm 1.7$ & $1.27 \pm 0.03$ \\
\hline
\end{tabular}




\begin{tabular}{llll}
\hline T-SiF3-20wt\% & $0.128 \pm 0.002$ & $4.1 \pm 1.5$ & $1.32 \pm 0.07$ \\
T-SiF3-25wt\% & $0.135 \pm 0.002$ & $2.8 \pm 1.3$ & $1.31 \pm 0.04$ \\
\hline
\end{tabular}

\subsection{Morphology}

The SEM images of silica aerogels with and without modification by $\mathrm{SiF}_{3}$ are presented in Figure 1. Secondary silica particles resolved to a scale of $100 \mathrm{~nm}$ are seen. It is apparent that the size of the particle aggregates did not change due to modification by $\mathrm{SiF}_{3}$ molecules. It is also apparent that the average particle sizes are similar in all cases. These observations are not surprising. First, in method 3, the silica networks were already formed by the time the $\mathrm{SiF}_{3}$ molecules were introduced. Second, no further reactions, including coarsening of silica particles, were possible once the residual $\mathrm{Si}-\mathrm{OH}$ groups were eliminated at a site. We now examine using SAXS data if the reactions with $\mathrm{SiF}_{3}$ molecules had any effect on the fractal structures of silica networks. 

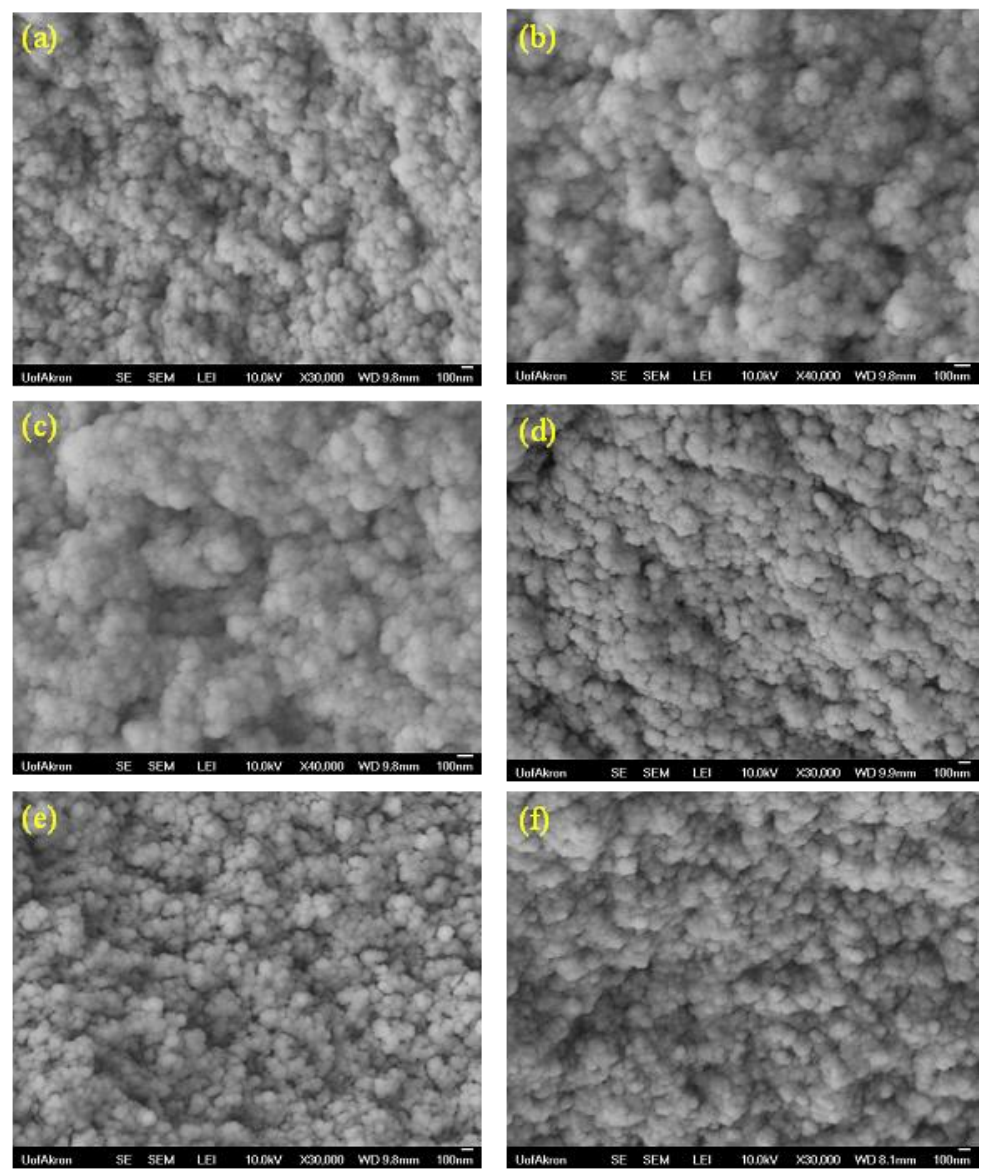

Figure 2. SEM images of aerogels without and with modification by SiF3. (a) T-0, (b) TSiF3-5wt\%, (c) T-SiF3-10wt\%, (d) T-SiF3-15wt\%, (e) T-SiF3-20wt\%, (f) T-SiF3-25wt\%.

The SAXS intensity $\boldsymbol{I}(\boldsymbol{q})$ plotted as a function of scattering vector $\boldsymbol{q}$ did not show distinct peaks as shown in Figure S2, indicating that the aerogels contained disordered structures. A plot of $\log \boldsymbol{I}(\boldsymbol{q})$ versus $\log \boldsymbol{q}$ (Figure 3) was used to obtain the value of fractal dimensions Ds following the method described in Duan et al. ${ }^{25}$. The intensity followed a power-law behaviour in the interval $0.04 \leq \boldsymbol{q} \leq 0.11 \AA^{-1}$ with exponents ranging from -3.37 to -3.42 . These correspond to fractal dimensions Ds in the range of $2.58-2.63$, as listed in Table 3 . A reduction of fractal dimension implies that the curved surface of the pore in baseline aerogel turned into smooth cylindrical pore. The power law was not satisfied for $\boldsymbol{q} \geq 0.11 \AA^{-1}$ because $\boldsymbol{q}$ became so large that the scattering process could resolve the structures of the size of individual atoms and the two-phase approximation was no longer satisfied. ${ }^{35}$ The values of 
the slope of the Porod's plot in the range of -4 to -3 , imply fractal nature of the silica networks. ${ }^{32}$ Close values of fractal dimension (Table 3 ) imply that very little change occurred in the silica networks after modification with $\mathrm{SiF}_{3}$ molecules, which agrees with the morphology shown in the SEM images. In this context, one wonders how $\mathrm{SiF}_{3}$ molecules positioned themselves in the aerogel structure such that the density and fractal networks remained unaffected.

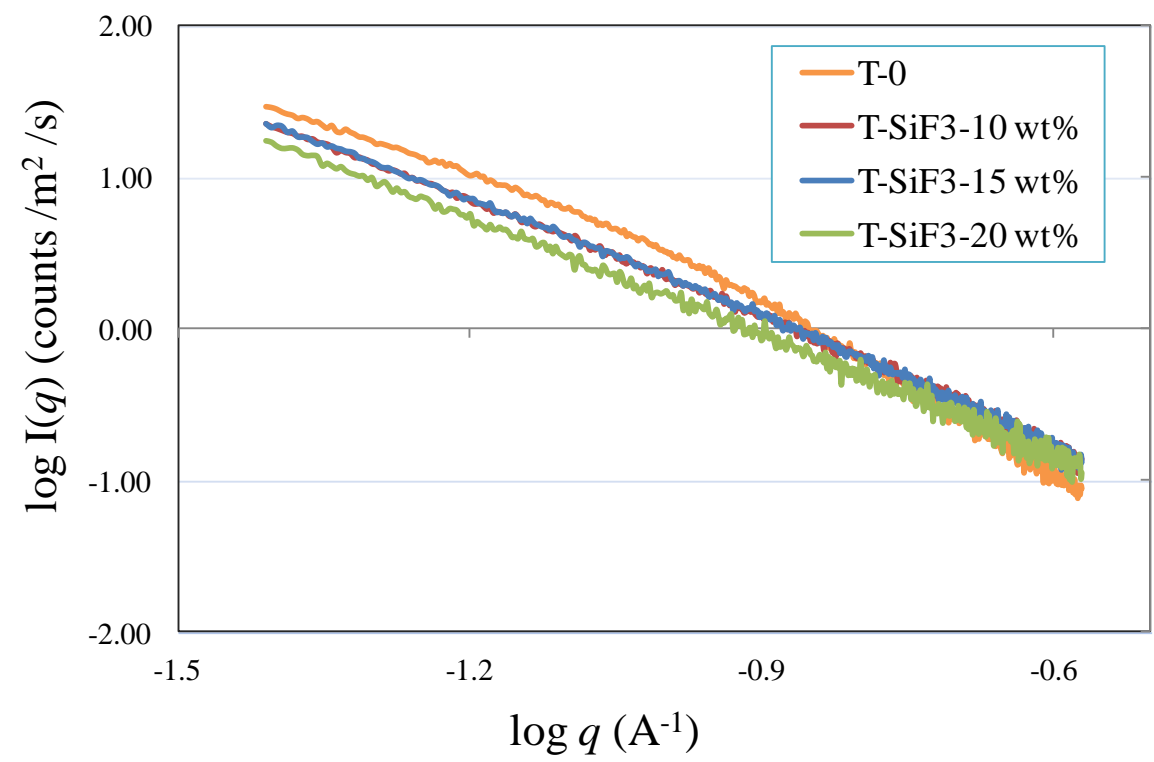

Figure 3. Porod's plot of $\mathrm{SiF}_{3}$-modified silica aerogels as a function of $q$ on a log-log scale.

Table 3. Slopes and fractal dimensions from Porod's plot of aerogels modified with $\mathrm{SiF}_{3}$.

\begin{tabular}{lcc}
\hline Sample & Slope & Ds \\
\hline T-0 & -3.41 & 2.59 \\
T-SiF3-10wt\% & -3.38 & 2.62 \\
T-SiF3-15wt\% & -3.42 & 2.58 \\
T-SiF3-20wt\% & -3.37 & 2.63 \\
\hline
\end{tabular}




\subsection{Structure of silica networks}

The ${ }^{29} \mathrm{Si}$ NMR spectrum of silica materials typically show several distinct peaks arising from $\mathrm{Si}(-\mathrm{OSi})_{2}(-\mathrm{OH})_{2}, \mathrm{Si}(-\mathrm{OSi})_{3}(-\mathrm{OH})$, and $\mathrm{Si}(-\mathrm{OSi})_{4}$ linkages. $^{36}$ These species are labelled respectively $\mathrm{Q}_{2}, \mathrm{Q}_{3}$, and $\mathrm{Q}_{4}$, with the peaks occurring more upfield with higher $\mathrm{Q}$ value. With the use of cross polarization, higher $\mathrm{Q}_{3}$ peak than $\mathrm{Q}_{4}$ peak maybe be displayed despite being almost fully condensed. This is attributed to the proximity of hydrogen atoms in hydroxyl groups to the silicon atoms in incompletely condensed silanes.

In an effort to better understand the chemical structures of silica particles in aerogels, the materials were characterized by ${ }^{13} \mathrm{C} \mathrm{NMR}$, with and without $\mathrm{SiF}_{3}$, and the data are shown in Figure 4. The peaks at $59 \mathrm{ppm}$ and $15 \mathrm{ppm}$ in ${ }^{13} \mathrm{C}$ NMR spectra from the unmodified aerogel (Figure 4a-d) arise from incompletely hydrolyzed ethoxy groups of TEOS. The level of remaining ethoxy groups is less than $5 \%$ of the starting material, as we reported previously. ${ }^{25}$ In $\mathrm{SiF}_{3}$-modified aerogels, the additional peaks from the $\mathrm{SiF}_{3}$ group occur at $-3.5 \mathrm{ppm}(\mathrm{Si}$ $\left.\mathrm{CH}_{3}\right), 8.0 \mathrm{ppm}\left(\mathrm{Si}-\mathrm{CH}_{2}-\right), 27 \mathrm{ppm}\left(-\mathrm{CH}_{2}-\right), 49 \mathrm{ppm}\left(\mathrm{Si}-\mathrm{OCH}_{3}\right)$, and $126 \mathrm{ppm}$ and $129 \mathrm{ppm}(-$ $\mathrm{CF}_{3}$ ), as seen in Figure 4(b-d). The presence of $-\mathrm{OCH}_{3}$ group in the ${ }^{13} \mathrm{C}$ spectra indicates that some of the $\mathrm{SiF}_{3}$ molecules were not completely hydrolyzed. The data shows that the amount of $\mathrm{SiF}_{3}$ present in the aerogels increased with an increase in the concentration of $\mathrm{SiF}_{3}$ molecules in the reaction mixture. The level of $\mathrm{SiF}_{3}$ molecules reacted onto silica networks was quantitatively evaluated using the data from ${ }^{29} \mathrm{Si}$ NMR. 


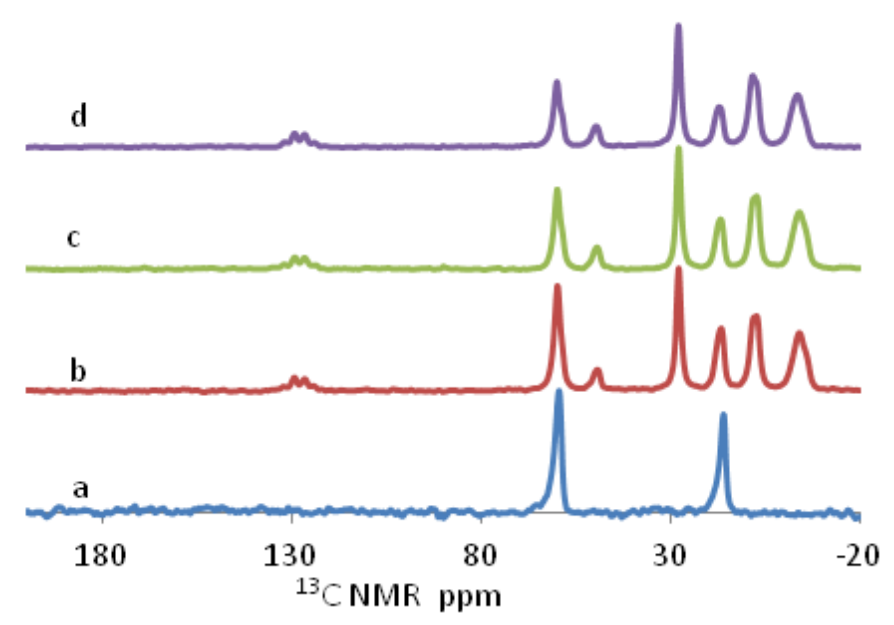

Figure 4. Solid-state ${ }^{13} \mathrm{C}$ NMR spectra of (a) T-0 (scans=13800) (b) T-SiF $35 w t \%$

(scans $=32768)$, (c) $\mathrm{T}-\mathrm{SiF}_{3} 10 \mathrm{wt} \%$ (scans=26760), and (d) $\mathrm{T}-\mathrm{SiF}_{3} 20 \mathrm{wt} \%$ (scans=28756). The cross-polarization time for all spectra is $3 \mathrm{~ms}$.

The product composition of $\mathrm{SiF}_{3}$-modified aerogels was inferred from the data of ${ }^{29} \mathrm{Si} \mathrm{NMR}$. The ${ }^{29} \mathrm{Si}$ NMR spectrum from the unmodified aerogel shows the presence of silicon sites from TEOS that has undergone different levels of crosslinking. The spectrum contains peaks at $-109 \mathrm{ppm}$ from $\mathrm{Q}_{4} \equiv \mathrm{Si}(-\mathrm{OSi})_{4}$, sites, $-102 \mathrm{ppm}$ from $\mathrm{Q}_{3} \equiv \mathrm{Si}(-\mathrm{OSi})_{3}(-\mathrm{OR} / \mathrm{H})$ sites and -89 ppm from $\mathrm{Q}_{2} \equiv \mathrm{Si}(-\mathrm{OSi})_{2}(-\mathrm{OR} / \mathrm{H})_{2}$ sites (Figure 5a). The larger number of $\mathrm{Q}_{2} / \mathrm{Q}_{3}$ sites in comparison to $\mathrm{Q}_{4}$ sites, indicating incomplete cross-linking, is consistent with the high surface area of TEOS aerogels. Upon reacting the aerogel with $\mathrm{SiF}_{3}$, the ${ }^{29} \mathrm{Si}$ spectrum contains two additional peaks at $-11 \mathrm{ppm}$ and, $-17 \mathrm{ppm}$ due to $\mathrm{Si}(-\mathrm{OSi})(-\mathrm{OR} / \mathrm{H})(-\mathrm{R})_{2}$ and $\mathrm{Si}(-$ $\mathrm{OSi})_{2}(-\mathrm{R})_{2}$ sites both originating from $\mathrm{SiF}_{3}$ (Figure $5 \mathrm{~b}$ ). In the $\mathrm{SiF}_{3}$-modified materials, the number of $\mathrm{Q}_{2} / \mathrm{Q}_{3}$ sites in the silica network is reduced while the number of $\mathrm{Q}_{4}$ sites increases (Figure 5b-d). The conversion of $\mathrm{Q}_{2} / \mathrm{Q}_{3}$ sites to $\mathrm{Q}_{4}$ sites is the result of the $\mathrm{SiF}_{3}$ molecules reacting with the $\mathrm{Si}-\mathrm{OH}$ groups of the aerogel network. The extent of reactions of $\mathrm{SiF}_{3}$ molecules to the silica network is still unknown. 
The extent of incorporation of $\mathrm{SiF}_{3}$ molecules into the silica network can be estimated from the ${ }^{29} \mathrm{Si}$ spectrum. However, as the data was collected while using the method of cross polarization $(\mathrm{CP})$, the signal intensity was dependent on the density of protons near each $\mathrm{Si}$ site. Thus, the intensity of the $\mathrm{Si}$ peak from $\mathrm{SiF}_{3}$, with a $\mathrm{CH}_{2}$ group bonded to $\mathrm{Si}$, would be larger than the signal from $\mathrm{Q}_{4}$ sites, which have a much lower density of nearby protons. The ratio of integrated peak values for $\mathrm{SiF}_{3}$ to silica network, for the sample with $5 \mathrm{wt} \% \mathrm{SiF}_{3}$, is $\sim 1: 2$. After correcting for the impact of $\mathrm{CP}$ on peak intensity, the actual ratio is $\sim 0.35: 1$, and consequently, $\sim 30-35 \%$ of the $\mathrm{Si}$ sites in the silica network had reacted with $\mathrm{SiF}_{3}$ molecules. This value increases for the samples exposed to 10 and $20 \% \mathrm{SiF}_{3}$ indicating 40 $45 \%$ of the sites reacted with $\mathrm{SiF}_{3}$ molecules. This last result shows that the silica system did appear to reach a saturation point in terms of reactions with $\mathrm{SiF}_{3}$ molecules, suggesting that more buried $\mathrm{Si}-\mathrm{OH}$ sites were not accessible to the $\mathrm{SiF}_{3}$ molecules. The ${ }^{29} \mathrm{Si}$ NMR spectrum from $\mathrm{SiF}_{3}$ only contains a peak at $-5 \mathrm{ppm}$, and the absence of this peak in the spectra from the $\mathrm{SiF}_{3}$-modified aerogels (Figure 5), reveals that the unreacted $\mathrm{SiF}_{3}$ was removed during the sample washing process.

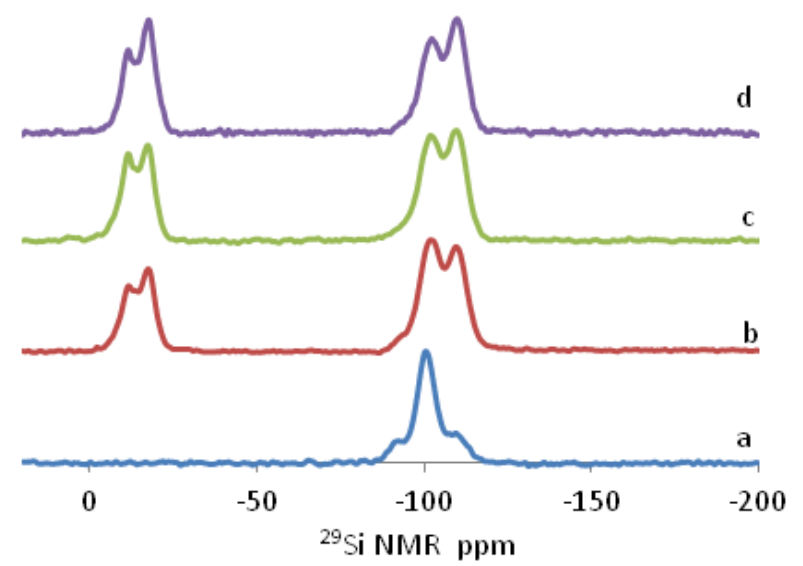

Figure 5. Solid-state ${ }^{29} \mathrm{Si}$ NMR spectra of (a) T-0 (scans=4908) (b) $\mathrm{T}-\mathrm{SiF}_{3} 5 \mathrm{wt} \%$ (scans=27268), (c) $\mathrm{T}-\mathrm{SiF}_{3} 10 \mathrm{wt} \%$ (scans=12964) and (d) $\mathrm{T}_{-} \mathrm{SiF}_{3} 20 \mathrm{wt} \%$ (scans=9596). 
We now revisit the insensitivity of the compressive modulus data reported in Table 2 to the concentration of $\mathrm{SiF} 3$ beyond $15 \mathrm{wt} \%$. As learned above from NMR data, such insensitivity is a direct ramification of the saturation of the degree of crosslinking of the silica networks by SiF3 molecules when SiF3 was used in the range of 10-20 wt $\%$.

\subsection{Surface properties}

\subsubsection{Surface area and pore size distribution from gas adsorption method}

The surface properties were studied by gas adsorption method and from the measurement of contact angles. The adsorption-desorption isotherms of aerogels without and with $\mathrm{SiF}_{3}$ modification are included in Figure 6. The adsorption isotherms showed the existence of micropores and mesopores, respectively from the initial adsorption curve and the hysteresis loop. The clearly visible hysteresis loop indicates the existence of a large amount of mesopores in the range of 2-50 $\mathrm{nm}$. The smaller hysteresis loop in the adsorption and desorption isotherms of unmodified aerogels indicates the existence of less mesopores. It is possible that the sizes of some of the pores are out of this range $(>50 \mathrm{~nm})$. This is also reflected from SEM images and from the lowest density among all aerogels. 


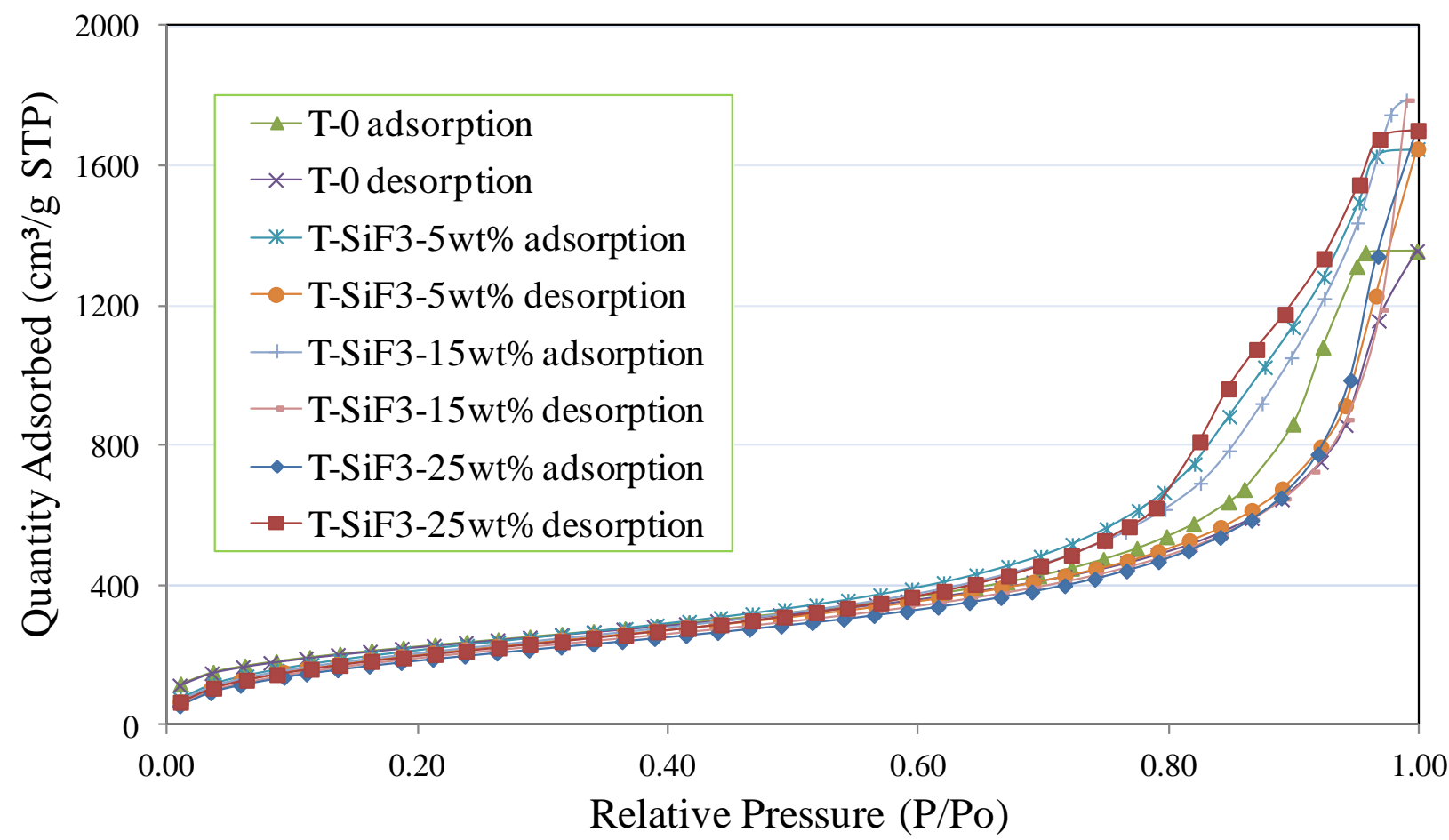

Figure 6. Adsorption and desorption isotherms of aerogels without/with modification by SiF3.

The pore size distributions of aerogels were obtained from desorption isotherms by the BJH method. Representative set of data are shown in Figure 7. The dominant pore size shifted left to smaller diameter for aerogels modified by $\mathrm{SiF}_{3}$ molecules. The peak corresponding to dominant pore size became more distinct due probably to a reduction of the portion of the micropores. This is also revealed by a reduction of micropore surface area shown in Table 4 . The BET surface area shows only small reduction after modification by $\mathrm{SiF}_{3}$ molecules. This small reduction in surface area can possibly be attributed to $\mathrm{SiF}_{3}$ segments blocking some of the micropores in the aerogels. The average pore size of aerogels with and without modification by $\mathrm{SiF}_{3}$ fell in the range of $10-13 \mathrm{~nm}$. The pore size showed small reduction indicating that only a coating of $\mathrm{SiF}_{3}$ molecules could form on the surfaces of the secondary 
particles. The results from gas adsorption method and NMR discussed earlier suggest that $\mathrm{SiF}_{3}$ molecules eliminated the residual $-\mathrm{OH}$ groups on the surfaces of secondary particles, forming thin coating layers.

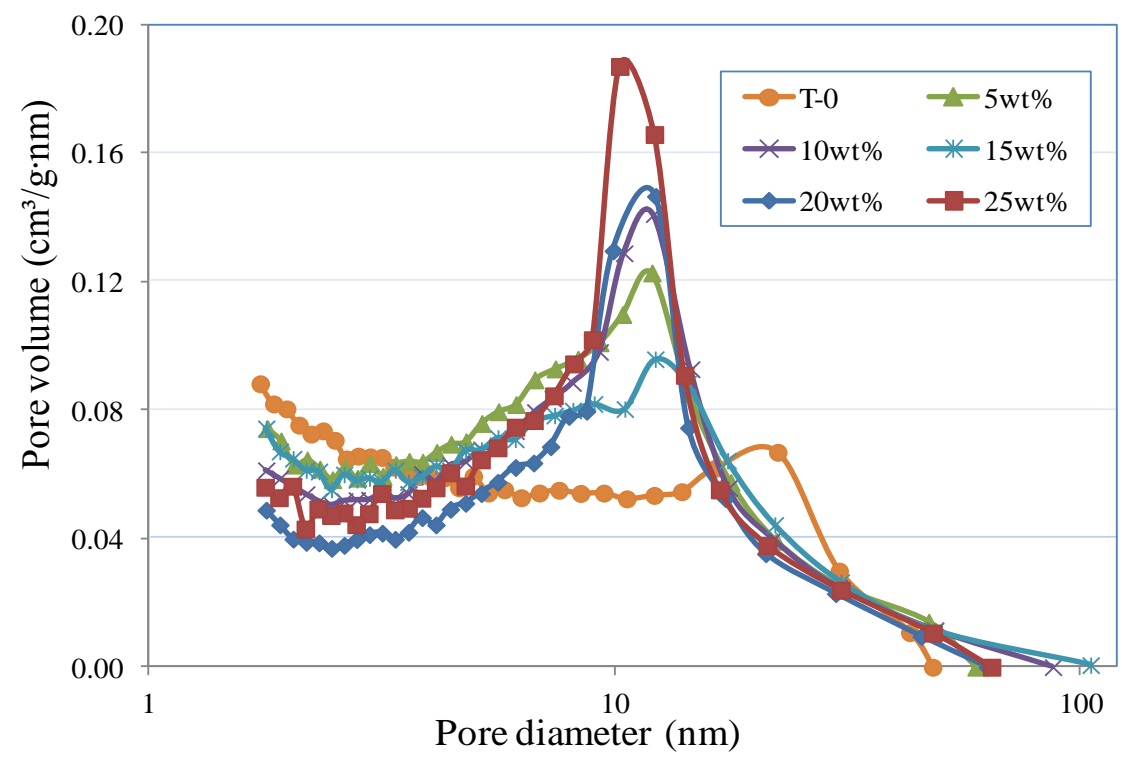

Figure 7. Pore size distribution of aerogels without/with modification by $\mathrm{SiF}_{3}$ obtained from desorption isotherm determined by BJH method.

Table 4. BET surface area, average pore size, and dominant pore size from gas adsorption method of TEOS aerogels without and with modification by $\mathrm{SiF}_{3}$

\begin{tabular}{ccccc}
\hline sample & $\begin{array}{c}\text { BET surface } \\
\text { area }\left(\mathrm{m}^{2} / \mathrm{g}\right)\end{array}$ & $\begin{array}{c}\text { Total pore } \\
\text { volume } \\
\left(\mathrm{cm}^{3} / \mathrm{g}\right)\end{array}$ & $\begin{array}{c}\text { Average pore } \\
\text { diameter from } \\
\text { BJH method }(\mathrm{nm})\end{array}$ & $\begin{array}{c}\text { Dominant pore } \\
\text { diameter } \\
(\mathrm{nm})\end{array}$ \\
\hline $\mathrm{T}-0$ & 804.7 & 2.54 & 12 & 22 \\
$\mathrm{~T}-\mathrm{SiF} 3-5 \mathrm{wt} \%$ & 790.3 & 2.55 & 12 & 12 \\
$\mathrm{~T}-\mathrm{SiF} 3-10 \mathrm{wt} \%$ & 752.6 & 2.85 & 11 & 12 \\
$\mathrm{~T}-\mathrm{SiF} 3-15 \mathrm{wt} \%$ & 756.8 & 2.76 & 11 & 12 \\
$\mathrm{~T}-\mathrm{SiF} 3-20 \mathrm{wt} \%$ & 733.0 & 2.26 & 11 & 12 \\
T-SiF3-25wt\% & 726.2 & 2.63 & 11 & 10 \\
\hline
\end{tabular}




\subsubsection{Contact angle}

It was seen earlier in the context of the data presented in Table 1 that the post-gelation modification strategy works better for rendering silica aerogels more hydrophobic. The water contact angle on compressed aerogel discs can reach greater than $150^{\circ}$ (Figure S3). The compressed disc of unmodified aerogel had a low contact angle value $\left(27.5^{\circ}\right)$ with deionized water. The aerogel specimens modified with 15 and $25 \mathrm{wt} \% \mathrm{SiF}_{3}$ show contact angle of $135^{\circ}$ and $154^{\circ}$ respectively. The contact angle data with methylene iodide are also presented in Table 5. Based on these contact angle data, the surface energy, including polar and dispersion components, and the value of polarity, were calculated as listed in Table 5. The polarity reduced from 0.53 for unmodified aerogels to 0.12 for aerogels treated with $10 \mathrm{wt} \% \mathrm{SiF}_{3}$ solution. The surface energy of modified aerogels with $\mathrm{SiF}_{3}$ concentration higher than 10 wt $\%$ was not calculated as the polarity reduced to almost zero. Figure $8(a)$ shows that a drop of water could sit on a monolithic aerogel without being absorbed. Figure 8(b) shows that a monolithic aerogel could float on water. The curvature of the water surface is clearly apparent in Figure 5(b), once again validating the hydrophobic nature of the aerogels.

Table 5. Contact angle on compressed aerogel discs. Modification by $\mathrm{SiF}_{3}$ was conducted by method 3. Polar $\left(\gamma_{p}\right)$ and dispersion $\left(\gamma_{d}\right)$ components of surface energy $\left(\gamma \equiv \gamma_{p}+\gamma_{d}\right)$ and polarity $\left(\gamma_{\mathrm{p}} / \gamma\right)$ are also presented.

\begin{tabular}{ccccccc}
\hline Specimen & $\begin{array}{c}\text { Contact angle } \\
\text { with } \mathrm{H}_{2} \mathrm{O}\end{array}$ & $\begin{array}{c}\text { Contact angle } \\
\text { with } \mathrm{CH}_{2} \mathrm{I}_{2}\end{array}$ & $\begin{array}{c}\gamma_{\mathrm{d}} ; \\
\mathrm{dyn} / \\
\mathrm{cm}\end{array}$ & $\begin{array}{c}\gamma_{\mathrm{p}} ; \\
\mathrm{dyn} / \\
\mathrm{cm}\end{array}$ & $\begin{array}{c}\gamma ; \\
\mathrm{dyn} / \mathrm{cm}\end{array}$ & $\gamma_{\mathrm{p}} / \gamma$ \\
\hline $\mathrm{T}-0$ & $27.3 \pm 0.4^{\circ}$ & $25.4 \pm 0.3^{\circ}$ & 31.8 & 36.6 & 68.4 & 0.54 \\
$\mathrm{~T}-\mathrm{SiF} 3-5 \mathrm{wt} \%$ & $70.5 \pm 0.9^{\circ}$ & $38.6 \pm 0.6^{\circ}$ & 30.4 & 14.8 & 45.3 & 0.33 \\
$\mathrm{~T}-\mathrm{SiF} 3-10 \mathrm{wt} \%$ & $106.1 \pm 0.6^{\circ}$ & $51.5 \pm 1.0^{\circ}$ & 30.4 & 4.3 & 34.8 & 0.12 \\
$\mathrm{~T}-\mathrm{SiF} 3-15 \mathrm{wt} \%$ & $134.7 \pm 1.0^{\circ}$ & $98.5 \pm 0.4^{\circ}$ & - & - & - & - \\
$\mathrm{T}-\mathrm{SiF} 3-20 \mathrm{wt} \%$ & $145.2 \pm 0.8^{\circ}$ & $108.1 \pm 0.5^{\circ}$ & - & - & - & - \\
$\mathrm{T}-\mathrm{SiF} 3-25 \mathrm{wt} \%$ & $154.1 \pm 0.5^{\circ}$ & $124.6 \pm 0.5^{\circ}$ & - & - & - & - \\
\hline
\end{tabular}



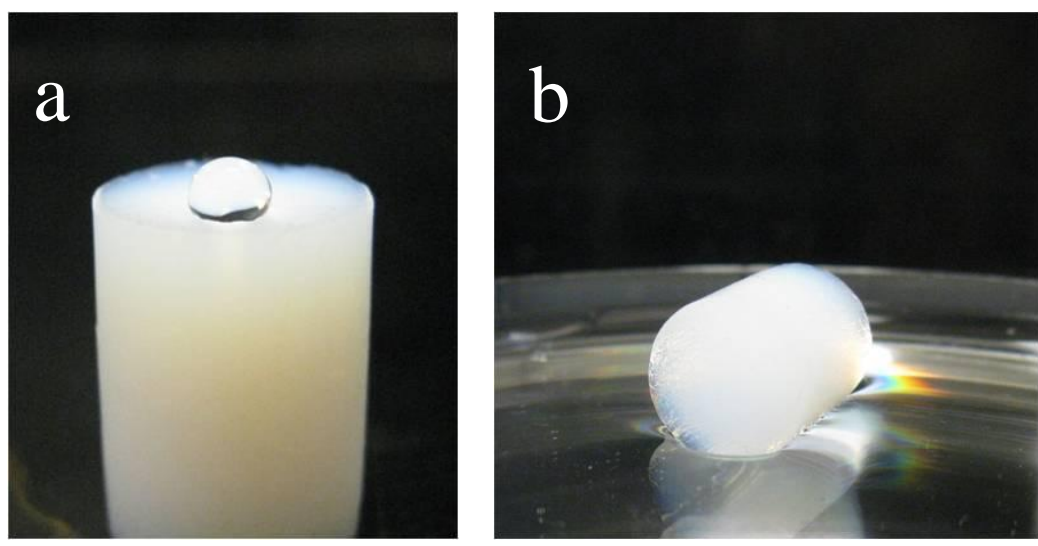

Figure 8. Images showing hydrophobic nature of T-SiF3-15wt\% aerogel. (a) A droplet of water sitting on top of the aerogel. (b) The aerogel monolith floats on top of water.

\section{Conclusions}

The study covered several schemes for surface modification of silica aerogels with $\mathrm{SiF}_{3}$ molecules. The post-gelation modification scheme in method 3 was found most effective in terms of changing the hydrophobic nature of the unmodified silica aerogels. One may argue if results comparable to method 3 can be obtained by using higher proportions of $\mathrm{SiF} 3$ in conjunction with method 1 . Based on the data presented in this study, we infer that the use of higher proportions of $\mathrm{SiF} 3$ in method 1 may produce inferior mechanical properties as bifunctional $\mathrm{SiF} 3$ molecules cannot produce enough crosslinked networks. The study considered formulation and fabrication process of some superhydrophobic aerogels, which are not commonly seen in literature. ${ }^{37-39}$ It is believed that the $\mathrm{SiF}_{3}$ molecules formed a monolayer coating on the surface of the secondary particles. Consequently, the surface area reduced only slightly, but the surface energy reduced significantly. Mechanical reinforcement was not significant at low concentration of $\mathrm{SiF}_{3}$ in solution, but the compressive modulus doubled when aerogels were modified with a $15 \mathrm{wt} \%$ solution of $\mathrm{SiF}_{3}$. 


\section{Acknowledgements}

We would like to express special thanks to Dr. Robert A. Weiss and Dr. Stephen Z.D. Cheng for allowing us using the contact angle goniometer and SAXS equipment. Financial assistantship from National Science Foundation in the form of award CMMI-1200484 is gratefully acknowledged.

\section{Notes and references}

1 M. A. Aegerter, N. Leventis, M. M. Koebel (Ed.), Aerogels Handbook, Springer, New York, 2011.

2 A. C. Pierre, A. Rigacci, $\mathrm{SiO}_{2}$ aerogels (Chapter II), in Aerogels Handbook, Springer, New York, 2011, 21-45.

3 A.V. Rao, Solid State Physics 1999, 41, 14.

4 G.S. Kim, S.H. Hyun, H.H. Park, J. Am. Ceram. Soc. 2001, 84, 453.

5 L.W. Hrubesh, L.E. Keene, V.R. Latorre, J. Mater. Res. 1993, 8, 1736.

6 J. Fricke, R. Caps, D. Buttner, U. Heinemann, E. Hummer, J. Non-Cryst. Solids 1987, 95-6, 1167.

7 G.M. Pajonk, J. Non-Cryst. Solids 1998, 225, 307.

8 A.V. Rao, G.M. Pajonk, D. Haranath, Mater. Sci. Technol. 2001, 17, 343.

9 M. Reim, G. Reichenauer, W. Korner, J. Manara, M. Arduini-Schuster, S. Korder, A. Beck, J. Fricke, J. Non-Cryst. Solids 2004, 350, 358.

10 Y.K. Akimov, Instruments and Experimental Techniques 2003, 46, 287.

11 Q. Yue, Y.Z. Li, M. Kong, J.C. Huang, X.J. Zhao, J. Liu, R.E. Williford, J Mater Chem 2011, 21, 12041.

12 J. Fricke, J. Non-Cryst. Solids 1992, 147, 356.

13 S.M. Jones, J. Sol-Gel Sci. Technol. 2006, 40, 351. 
14 M.L. Anderson, D.R. Rolison, C.I. Merzbacher, Engineered Nanostructural Films and Materials 1999, 3790, 38.

15 S. Martinez, M. Moreno-Manas, A. Vallribera, U. Schubert, A. Roig, E. Molins, New J. Chem. 2006, 30, 1093.

16 J.L. Gurav, I.K. Jung, H.H. Park, E.S. Kang, D.Y. Nadargi, Journal of Nanomaterials 2010, Article ID 409310, 11 pages, doi:10.1155/2010/409310.

17 A.J. Salinas, M. Vallet-Regi, J.A. Toledo-Fernandez, R. Mendoza-Serna, M. Pinero, L. Esquivias, J. Ramirez-Castellanos, J.M. Gonzalez-Calbet, Chem. Mater. 2009, 21, 41.

18 S. Smitha, P. Shajesh, P. Mukundan, T.D.R. Nair, K.G.K. Warrier, Collioids Surf. B. Biointerfaces 2007, 55, 38.

19 G. Zhang, A. Dass, A.M. Rawashdeh, J. Thomas, J.A. Counsil, C. Sotiriou-Leventis, E.F. Fabrizio, F. Ilhan, P. Vassilaras, D.A. Scheiman, L. McCorkle, A. Palczer, J.C. Johnston, M.A. Meador, N. Leventis, J. Non-Cryst. Solids 2004, 350, 152.

20 M.A.B. Meador, L. A. C., Process for preparing polymer reinforced silica aerogels $U S$ patent 8,067,478 2011.

21 K.A.D. Obrey, K.V. Wilson, D.A. Loy, J Non-Cryst Solids 2011, 357, 3435.

22 J.P. Randall, M.A.B. Meador, Jana, S.C. Jana, ACS Applied Materials \& Interfaces $2011,3,613$.

23 N. Leventis, C. Sotiriou-Leventis, G.H. Zhang, A.M.M. Rawashdeh, Nano Lett. 2002, $2,957$.

24 S. Mulik, C. Sotiriou-Leventis, G. Churu, H.B. Lu, N. Leventis, Chem Mater 2008, 20, 5035 .

25 Y. Duan, S.C. Jana, A.M. Reinsel, B. Lama, M.P. Espe, Langmuir 2012, 28, 15362.

26 Y. Duan, S.C. Jana, B. Lama, M.P. Espe, Langmuir 2013, 29, 6156. 
27 A.V. Rao, M.M. Kulkarni, D.P. Amalnerkar, T. Seth, Appl Surf Sci 2003, 206, 262.

28 M.L. Liu, D.A. Yang, Y.F. Qu, J. Non-Cryst. Solids 2008, 354, 4927.

29 A. M. Anderson, M. K. Carroll, Hydrophobic silica aerogels: Review of synthesis, properties and applications. In Aerogels Handbook, Springer, New York, 2011, 4777.

30 C. Bonhomme, C. Coelho, N. Baccile, C. Gervais, T. Azais, F. Babonneau, Accounts Chem Res 2007, 40, 738.

31 A. Craievich, M.A. Aegerter, D.I. Dossantos, T. Woignier, J. Zarzycki, J Non-Cryst Solids 1986, 86, 394.

32 H.D. Bale, P.W. Schmidt, Phys. Rev. Lett. 1984, 53, 596.

33 D.W. Schaefer, K.D. Keefer, Phys. Rev. Lett. 1984, 53, 1383.

34 D.W. Schaefer, K.D. Keefer, Phys. Rev. Lett. 1986, 56, 2199.

35 K. Sinko, V. Torma, A. Kovacs, J. Non-Cryst. Solids 2008, 354, 5466.

36 E. Lippmaa, M. Magi, A. Samoson, G. Engelhardt, A.R. Grimmer, J. Am. Chem. Soc. 1980, 102, 4889.

37 P.B. Wagh, S.V. Ingale, S.C. Gupta, J. Sol-Gel Sci. Technol. 2010, 55, 73.

38 A.P Rao, A.V. Rao, J Mater Sci 2010, 45, 51.

39Z.S. Dou, H. Xu, Y.J. Cui, G.D. Qian, Rare Metal Mat Eng 2010, 39, 401. 